Kansas State University Libraries

New Prairie Press

\title{
RELATIVE POTENCY ESTIMATION IN DIRECT BIOASSAY WITH MEASUREMENT ERRORS*
}

Weixing Song

Follow this and additional works at: https://newprairiepress.org/agstatconference

Part of the Agriculture Commons, and the Applied Statistics Commons

\section{(c) $(1) \ominus$}

This work is licensed under a Creative Commons Attribution-Noncommercial-No Derivative Works 4.0 License.

\section{Recommended Citation}

Song, Weixing (2009). "RELATIVE POTENCY ESTIMATION IN DIRECT BIOASSAY WITH MEASUREMENT ERRORS*," Conference on Applied Statistics in Agriculture. https://doi.org/10.4148/2475-7772.1075

This is brought to you for free and open access by the Conferences at New Prairie Press. It has been accepted for inclusion in Conference on Applied Statistics in Agriculture by an authorized administrator of New Prairie Press. For more information, please contact cads@k-state.edu. 


\title{
RELATIVE POTENCY ESTIMATION IN DIRECT BIOASSAY WITH MEASUREMENT ERRORS*
}

\author{
WEIXING SONG
}

\begin{abstract}
The dosage levels measured in direct bioassays are often contaminated with measurement errors, which are usually neglected in the statistical inference. This paper proposes several estimation procedures for the relative potencies in direct bioassays taking the measurement errors into account. Asymptotic theories are developed for constructing the confidence intervals. Numerical simulations are also included to compare different estimation procedures.
\end{abstract}

Key words: Relative Potency; Bioassay; Errors-in-Variables Model; Deconvolution Kernel Estimator; Simulation Extrapolation; Empirical Likelihood.

\section{INTRODUCTION}

The relative potency of a test preparation $T$ compared to a standard preparation $S$ in the direct bioassay is defined by the ratio $\rho=\mu_{s} / \mu_{t}$, where $\mu_{s}$ is the population mean of the IED (individual effective dose) from standard preparation treated group and $\mu_{t}$ is the one from the test preparation treated group. See Finney (1978), Hubert (1992), and Govindarajulu (2001) for more discussion on the direct bioassay. A commonly used estimator for $\rho$ is $R=\bar{X}_{s} / \bar{X}_{t}$, where $\bar{X}$ is the sample mean, the subscripts indicating the different preparations. If the observed IED from both preparations follow normal distributions with the same variance, Fieller's theorem can be applied to construct a confidence interval for $\rho$. But the IED obtained in the bioassay often has some systematic or random measurement error. For example, if two stimuli of unequal potency are applied at equal rates, subjects receiving the less potent stimulus will have longer average times under treatment than those receiving the more potent. The time-lag will bias the comparison of the preparations. Even if the timelag does not exist, it is difficult to ensure that subjects will receive exactly the right dose to produce the desired response, since the doses applied to the subject may be affected by the time, experimenter's skill, and other unidentified random factors. The systematic measurement error can be tackled with nonstatistical methods, hence we will mainly deal with the random measurement errors. For convenience, in the sequel, we will use $x, X$ to denote the true and measured responses from the standard preparation, and $y, Y$ the responses from the

\footnotetext{
*The research is supported by USRG No.2232 of Kansas State University

Weixing Song is an Assistant Professor in the Department of Statistics, Kansas State University, Manhattan, KS, 66502, USA (e-mail weixing@ksu.edu).
} 
test preparation. Accordingly, $\mu_{x}$ is used to denote the population mean of the IED for the standard preparation, and $\mu_{y}$ for test preparation.

Let $(x, y)$ be the true doses from two preparations in the direct bioassay. Because of the measurement errors, one can not really observe $(x, y)$. Instead, a surrogate $(X, Y)$ can be obtained. The surrogate $(X, Y)$ and the true doses $(x, y)$ are related in certain ways. Modeling the relationship between $X$ and $x, Y$ and $y$ is a major topic in measurement error studies. The simplest and also the most commonly seen case is the following additivity structure,

$$
X=x+u, \quad Y=y+v,
$$

where $u$ and $v$ are measurement errors. See Fuller (1987), Carroll, Ruppert and Stefanski (1995) for a detailed discussion on this case and more complicated formulations. The independence of $x$ and $u, y$ and $v$ are often set as a precondition. Moreover, in practice, the two preparations are also assumed to be independent, or $(x, u)$ and $(y, v)$ are independent. Clearly we can not estimate $\rho$ from the unobserved $(x, y)$. But if $E u=E v=0$, one can see that $E X=E x$ and $E Y=E y$, this implies we can estimate $\rho$ by the ratio of sample means from the observations $X$ and $Y$. Note that $X$ and $Y$ are more variable than $x$ and $y$, respectively, if the measurement errors $u$ and $v$ have positive variances. As a consequence, the estimator for $\rho$ based on the surrogates will be less precise, and the corresponding confidence interval for $\rho$ will be wider. The current research will propose several estimation procedures in which the information from measurement errors will be taken into account in constructing the estimator and the confidence intervals of $\rho$.

In this paper, we will assume that the density functions of the measurement errors $u$ and $v$ are known. This is a strict but quite common assumption in the literature of measurement error modeling. In some cases, when a validation data set is available, one can estimate the density functions, hence some numeric characteristic associated with the densities, by some well known nonparametric smoothing techniques, such as the kernel, regression splines and so on. Under some conditions, in particular, if the sample size in the validation data set is larger than the one in the main data set, then all the large sample theories developed in this paper are still valid after replacing the quantities related to the measurement errors by their parametric or nonparametric estimators.

The paper is organized as follows. The naive estimator will be introduced in Section 2. The bias-attenuation estimator will be discussed in Section 3, together with the Fiellertype confidence interval under the normality assumptions. Some nonparametric estimation procedures will be discussed in Section 4, including the SIMEX estimator, deconvolution kernel estimator, empirical likelihood estimator and the SIMEX-Empirical likelihood estimators. The simulation studies will be given in Section 5 where various estimation procedures are compared. We also made some recommendations there based on the simulation results. 


\section{NAIVE ESTIMATOR}

The naive estimation procedure simply treats the surrogates $(X, Y)$ 's as the true doses $(x, y)$. Then the relative potency $\rho$ is estimated by the ratio $R=\bar{X}_{m} / \bar{Y}_{n}$, where $\bar{X}_{m}$ is the sample mean of $X_{1}, X_{2}, \ldots, X_{m}$ which are generated from the first relation in model (1.1), and $\bar{Y}_{n}$ is similarly constructed except the data are obtained from the second relation in model (1.1). If

(a) $x$ and $y$ are normally distributed with the same variance, say $\sigma_{d}^{2}$,

(b) $u$ and $v$ are normally distributed with means 0 , and the same variances, say $\sigma_{e}^{2}$,

then a Fieller-type confidence interval with confidence level $1-\alpha$ is given by

$$
\frac{1}{1-g}\left(R \pm \frac{t S}{\bar{Y}_{n}} \sqrt{\frac{1-g}{m}+\frac{R^{2}}{n}}\right),
$$

where $S^{2}$ is the pooled variance of the two samples $X_{1}, \ldots, X_{m}, Y_{1}, \ldots, Y_{n}, t$ is the $1-\alpha / 2$ percentile of a $t$-distribution with degrees of freedom $m+n-2$, and $g=t^{2} S^{2} /\left(n \bar{Y}_{n}^{2}\right)$. See Fieller, E.C. (1932) and Hubert, J.J. (1992) for the derivation of (2.1).

The normality and the homoscedasticity of $x$ and $y, u$ and $v$ are too restricted for some direct bioassays. They can be removed by resorting to large sample theory. In fact, one can show that

Theorem 2.1 Suppose $m / n \rightarrow \lambda \geq 0$ as $m, n \rightarrow \infty$. Then $\sqrt{m}\left(R-\rho_{0}\right) \Longrightarrow N\left(0, \sigma_{\rho}^{2}\right)$, where

$$
\sigma_{\rho}^{2}=\frac{\operatorname{Var}(X)}{E^{2}(Y)}+\frac{\lambda E^{2}(X) \operatorname{Var}(Y)}{E^{4}(Y)} .
$$

The proof of this theorem is a simple application of Delta-method, see p.45 in Ferguson (1996). Let $\hat{\sigma}_{\rho}^{2}$ be any consistent estimator of $\sigma_{\rho}^{2}$. Then a confidence interval for $\rho$ with confidence level $1-\alpha$ is given by $R \pm z_{1-\alpha / 2} \hat{\sigma}_{\rho}$, where $z_{1-\alpha / 2}$ is the $1-\alpha / 2$ percentile of standard normal distribution. A simple consistent estimator of $\sigma_{\rho}^{2}$ can be obtained by replacing $E(X)$ with $\bar{X}_{m}, E(Y)$ with $\bar{Y}_{m}, \operatorname{Var}(X)$ with the sample variance of $X_{i}$ 's, and $\operatorname{Var}(Y)$ with the sample variance of $Y_{j}$ 's. $\lambda$ is estimated with $m / n$. The naive point estimator of $\rho$ is less precise because of the larger variance of the surrogates $X$ and $Y$. As a consequence, the naive confidence interval will be wider when the measurement errors have bigger variabilities. This point will become clearer in the next section.

\section{BIAS ATTENUATION ESTIMATOR}

Since $X$ has a larger variance than $E(x \mid X)$, the conditional expectation of $x$ given $X$, so it would be desirable, at least intuitively, to use $E(x \mid X)$ instead of using $X$, if $E(x \mid X)$ has an 
explicit expression. This technique is called regression calibration, see Carroll et al. (1995). Suppose for the the time being that the conditions (a) and (b) in Section 2 hold, then

$$
E(x \mid X)=\mu_{x}+\frac{\sigma_{d}^{2}}{\sigma_{d}^{2}+\sigma_{e}^{2}}\left(X-\mu_{x}\right)=\frac{\sigma_{d}^{2}}{\sigma_{d}^{2}+\sigma_{e}^{2}} X+\frac{\sigma_{e}^{2}}{\sigma_{d}^{2}+\sigma_{e}^{2}} \mu_{x}
$$

which also has a normal distribution with mean $\mu_{X}$, and variance $\sigma_{d}^{4} /\left(\sigma_{d}^{2}+\sigma_{e}^{2}\right)$. it is easy to see that $\sigma_{d}^{4} /\left(\sigma_{d}^{2}+\sigma_{e}^{2}\right)$ is less than the variance of $x$, hence the variance of $X$. Denote $\widetilde{X}_{m}=\sum_{i=1}^{m} E\left(x_{i} \mid X_{i}\right) / m$, similarly $\widetilde{Y}_{n}=\sum_{j=1}^{n} E\left(y_{j} \mid Y_{j}\right) / n$. One can estimate $\rho$ by $\widetilde{R}=\widetilde{X}_{m} / \widetilde{Y}_{n}$. Note that,

$$
\widetilde{X}_{m}=\frac{\sigma_{d}^{2}}{\sigma_{d}^{2}+\sigma_{e}^{2}} \bar{X}_{m}+\frac{\sigma_{e}^{2}}{\sigma_{d}^{2}+\sigma_{e}^{2}} \mu_{x}, \quad \widetilde{Y}_{n}=\frac{\sigma_{d}^{2}}{\sigma_{d}^{2}+\sigma_{e}^{2}} \bar{Y}_{n}+\frac{\sigma_{e}^{2}}{\sigma_{d}^{2}+\sigma_{e}^{2}} \mu_{y}
$$

and

$$
\begin{aligned}
& \operatorname{Var}\left(\widetilde{X}_{m}\right)=\frac{\sigma_{d}^{4}}{\left(\sigma_{d}^{2}+\sigma_{e}^{2}\right)^{2}} \frac{\sigma_{d}^{2}+\sigma_{e}^{2}}{m}=\frac{\sigma_{d}^{4}}{m\left(\sigma_{d}^{2}+\sigma_{e}^{2}\right)}, \\
& \operatorname{Var}\left(\widetilde{Y}_{n}\right)=\frac{\sigma_{d}^{4}}{\left(\sigma_{d}^{2}+\sigma_{e}^{2}\right)^{2}} \frac{\sigma_{d}^{2}+\sigma_{e}^{2}}{n}=\frac{\sigma_{d}^{4}}{n\left(\sigma_{d}^{2}+\sigma_{e}^{2}\right)} .
\end{aligned}
$$

Therefore, a confidence interval for $\rho$ can be constructed by applying Fieller's theorem to $\widetilde{R}=\widetilde{X}_{m} / \widetilde{Y}_{n}$. Unfortunately, $\widetilde{X}_{m}$ and $\widetilde{Y}_{n}$ depend on some unknown quantities like $\mu_{x}, \mu_{y}$ and $\sigma_{d}^{2}$. An ad-hoc plug-in method is to replace $\mu_{x}$ with $\bar{X}_{m}, \mu_{y}$ with $\bar{Y}_{n}$, then $\widetilde{X}_{m}$ and $\widetilde{Y}_{n}$ will reduce to $\bar{X}_{m}$ and $\bar{Y}_{n}$ respectively, accordingly $\widetilde{R}$ reduces to $R=\bar{X}_{m} / \bar{Y}_{n}$. Finally, we can obtain a confidence interval for $\rho$ which is similar to the naive one (2.1) except for $S^{2}$ now being replaced by an estimator of $\sigma_{d}^{4} /\left(\sigma_{d}^{2}+\sigma_{e}^{2}\right)$. A good estimator for $\sigma_{d}^{2}$ can be chosen as

$$
\hat{\sigma}_{d}^{2}=\frac{(m-1) S_{x}^{2}+(n-1) S_{y}^{2}}{m+n-2}-\sigma_{e}^{2},
$$

so a natural estimator for $\sigma_{d}^{4} /\left(\sigma_{d}^{2}+\sigma_{e}^{2}\right)$ will be $\tilde{S}^{2}=\left(S^{2}-\sigma_{e}^{2}\right)^{2} / S^{2}$, where $S^{2}$ is the same as in the naive case. This implies the bias attenuated confidence interval has the form of

$$
\frac{1}{1-\tilde{g}}\left(R \pm \frac{t \tilde{S}}{\bar{Y}_{n}} \sqrt{\frac{1-\tilde{g}}{m}+\frac{R^{2}}{n}}\right),
$$

where $\tilde{g}=t^{2} \tilde{S}^{2} /\left(n \bar{Y}_{n}^{2}\right), t$ is the same as in (2.1).

One can easily tell that the confidence interval (3.2) is far from satisfying, the actual confidence level may not be close to the nominal level, since so many unknown quantities are replaced by their estimators. Nevertheless, since all estimator plugged in are consistent, we can imagine the actual confidence level would be close to the nominal level $1-\alpha$ as the sample size gets bigger.

Now it is the time to consider which confidence estimator has the shortest length. Denote $L_{1}$ as the length of (2.1), and $L_{2}$ as the length of (3.2). In fact, we have

$$
L_{1}=\frac{2 t S}{(1-g) \bar{Y}_{n}} \sqrt{\frac{1-g}{m}+\frac{R^{2}}{n}}, \text { and } L_{2}=\frac{2 t \tilde{S}}{(1-\tilde{g}) \bar{Y}_{n}} \sqrt{\frac{1-\tilde{g}}{m}+\frac{R^{2}}{n}} .
$$


It amounts to compare

$$
\frac{S}{1-g} \sqrt{\frac{1-g}{m}+\frac{R^{2}}{n}}, \text { and } \frac{\tilde{S}}{1-\tilde{g}} \sqrt{\frac{1-\tilde{g}}{m}+\frac{R^{2}}{n}}
$$

Since $\tilde{S}^{2} \leq S^{2}$ and $\tilde{g} \leq g$, so it is easy to see that $L_{1} \geq L_{2}$, equality holds if and only if $\sigma_{e}^{2}=0$, or the data have no measurement errors.

A large sample confidence interval does not need the conditions (a) and (b) in Section 2 , but it does require the knowledge about the variances of measurement errors, although the variances may not be the same.

Since

$$
\sqrt{m}\left(\tilde{X}_{m}-\mu_{x}\right) \Longrightarrow N\left(0, \sigma_{x}^{4} /\left(\sigma_{x}^{2}+\sigma_{u}^{2}\right)\right), \quad \sqrt{n}\left(\tilde{Y}_{n}-\mu_{y}\right) \Longrightarrow N\left(0, \sigma_{y}^{4} /\left(\sigma_{y}^{2}+\sigma_{v}^{2}\right)\right),
$$

and these two preparations are independent, so we can prove the following theorem.

Theorem 3.1 Suppose $m / n \rightarrow \lambda \geq 0$ as $m, n \rightarrow \infty$. Then $\sqrt{m}\left(R-\rho_{0}\right) \Longrightarrow N\left(0, \tilde{\sigma}_{\rho}^{2}\right)$, where

$$
\tilde{\sigma}_{\rho}^{2}=\frac{\sigma_{x}^{4}}{\left(\sigma_{x}^{2}+\sigma_{u}^{2}\right) E^{2}(Y)}+\frac{\lambda E^{2}(X) \sigma_{y}^{4}}{\left(\sigma_{y}^{2}+\sigma_{v}^{2}\right) E^{4}(Y)}
$$

Let $\hat{\sigma}_{\rho}^{2}$ be any consistent estimator of $\tilde{\sigma}_{\rho}^{2}$. Then a confidence interval for $\rho$ with confidence level $1-\alpha$ is given by $R \pm z_{1-\alpha / 2} \hat{\tilde{\sigma}}_{\rho}$, where $z_{1-\alpha / 2}$ is the $1-\alpha$ percentile of standard normal distribution. A simple consistent estimator of $\sigma_{\rho}^{2}$ can be obtained by replacing $E(X)$ with $\bar{X}_{m}, E(Y)$ with $\bar{Y}_{m}, \sigma_{x}^{2}$ with $S_{X}^{2}-\sigma_{u}^{2}$, and $\sigma_{y}^{2}$ with $S_{Y}^{2}-\sigma_{v}^{2}$, where $S_{X}^{2}$ and $S_{Y}^{2}$ are the sample variance of $X$ 's and $Y$ 's, respectively. Again, the actual confidence level based on this asymptotic theory may not be equal to the nominal level.

Remark: In some cases, $\kappa_{x}=\sigma_{d}^{2} / \sigma_{X}^{2}, \kappa_{y}=\sigma_{d}^{2} / \sigma_{Y}^{2}$, also called reliability ratio, or heritability, are available. Then one may incorporate this knowledge to estimate the relative potency. In fact, one may simply replace $\tilde{S}^{2}$ by $\kappa^{2} S^{2}$ in (3.2) if $\kappa_{x}=\kappa_{y}$, where $S^{2}$ is the pooled sample variance using the data $X$ 's and $Y$ 's. Accordingly, the asymptotic variance of $R$ in Theorem 3.1 can be estimated by $\kappa_{x} S_{X}^{2} / \bar{Y}_{n}^{2}+\lambda \kappa_{y} \bar{X}_{m}^{2} / \bar{Y}_{n}^{4}$.

\section{NONPARAMETRIC METHODS}

This section presents several nonparametric methods, some of them can take care of the measurement errors directly, so a shorter confidence interval can be obtained. However, some of these methods do not correct the bias, such as the empirical likelihood estimator. But some modifications of these methods can lead to a procedure which can eliminate the measurement error. 


\subsection{SIMEX ESTIMATOR}

The SIMEX (simulation extrapolation) procedure was proposed by Cook and Stefanski (1995) and further developed by Carroll, et al. (1996) and Stefanski and Cook (1995). It is obtained by adding extra noise to the original observations, then building a trend of the estimator, obtained by pretending there is no measurement error, versus the variance of the added noises, and extrapolating this trend back to the case of no measurement error. As Stefanski and Cook (1995) point out, SIMEX is a complete method for estimating the function of $\mu$ from $N\left(\mu, \sigma^{2}\right)$ with $\sigma^{2}$ known or independently estimated by some estimator. SIMEX also works very well for other symmetric distribution families, though theory is lacking.

Let us begin with the point estimator for $\rho$, the ratio of sample means. We shall present two SIMEX procedures, both take advantage of the availability of the distribution of the measurement errors and have two steps.

\section{SIMEX Procedure 1:}

- Simulation step: For $\lambda \geq 0$, generate an i.i.d. sample of size $m$ from the distribution of $u$, say $\tilde{u}_{i}, i=1,2, \ldots, m$, calculate $\tilde{X}_{i}=X_{i}+\sqrt{\lambda} \tilde{u}_{i}$ and the average of $\tilde{X}_{i}$ 's. Repeat this step $B$ times to obtain $B$ averages, then compute the mean of these averages. Write this mean as $\tilde{X}(\lambda)$. Redo the above step by using different $\lambda \geq 0$. Suppose we are selecting $\lambda=\lambda_{1}, \lambda_{2}, \ldots, \lambda_{k}$. Then we obtain $\tilde{X}\left(\lambda_{1}\right), \tilde{X}\left(\lambda_{2}\right), \ldots, \tilde{X}\left(\lambda_{k}\right)$. Finally, using a linear, quadratic, or other proper curves to fit the data set $(\lambda, \tilde{X}(\lambda))$.

- Extrapolation step: Extrapolate the fitted curve to $\lambda=-1$. Denote the fitted value as $\tilde{X}(-1)$.

Repeat the above procedure for the observations from $Y$ with $m$ replaced by $n$. Denote the fitted value as $\tilde{Y}(-1)$. Then the SIMEX point estimator of $\rho$ is given be the ratio

$$
\hat{\rho}=\tilde{X}(-1) / \tilde{Y}(-1) \text {. }
$$

The technical reason for using $\lambda=-1$ in the extrapolation step can be find in Cook and Stefanski (1995). The other modification for the above SIMEX procedure is

\section{SIMEX Procedure 2:}

- Simulation step: For $\lambda \geq 0$, generate an i.i.d. sample of size $m$ from the distribution of $u$, say $\tilde{u}_{i}, i=1,2, \ldots, m$, calculate $\tilde{X}_{i}=X_{i}+\sqrt{\lambda} \tilde{u}_{i}$ and the average of $\tilde{X}_{i}$ 's. Repeat this step $B$ times to obtain $B$ averages, then compute the mean of these averages. Write this mean as $\tilde{X}(\lambda)$. Redo the above step by using different $\lambda \geq 0$. Suppose we are selecting $\lambda=\lambda_{1}, \lambda_{2}, \ldots, \lambda_{k}$. Then we obtain $\tilde{X}\left(\lambda_{1}\right), \tilde{X}\left(\lambda_{2}\right), \ldots, \tilde{X}\left(\lambda_{k}\right)$. Repeat the above procedure for the observations from $Y$ with $m$ replaced by $n$. Suppose we obtain $\tilde{Y}\left(\lambda_{1}\right), \tilde{Y}\left(\lambda_{2}\right), \ldots, \tilde{Y}\left(\lambda_{k}\right)$. Then calculate the ratios $\tilde{X}\left(\lambda_{1}\right) / \tilde{Y}\left(\lambda_{1}\right), \tilde{X}\left(\lambda_{2}\right) / \tilde{Y}\left(\lambda_{2}\right)$, 
$\ldots, \tilde{X}\left(\lambda_{k}\right) / \tilde{Y}\left(\lambda_{k}\right)$. Finally, using a quadratic, cubic or other proper curves to fit the data set $(\lambda, \tilde{X}(\lambda) / \tilde{Y}(\lambda))$.

- Extrapolation step: Extrapolate the fitted curve to $\lambda=-1$. The fitted value at $\lambda=-1$ will be the estimate for the relative potency.

A SIMEX confidence interval for $\rho$ can be constructed based on any existing confidence interval from the true data set. For example, the naive confidence interval (2.1) depends on $R, \bar{Y}_{n}$ and $S$, simply replacing these quantities by their SIMEX estimators will leads to a SIMEX confidence interval.

In addition to the linear and quadratic extrapolants, a nonlinear extrapolation is based on the model $f(\lambda)=a+b /(c+\lambda)$. As mentioned in the seminal paper by Cook and Stefanski (1994), when the measurement error is normally distributed, each of these three extrapolation functions is exact for certain estimators. But in practice, one can decide the proper extrapolation function by looking at the scatter plots of $(\lambda, \tilde{X}(\lambda)),(\lambda, \tilde{Y}(\lambda))$, or $(\lambda, \tilde{X}(\lambda) / \tilde{Y}(\lambda))$. For the values of $\lambda$ used in the SIMEX procedure, Cook and Stefanski (1994) suggest selecting values between 0 and 2 .

\subsection{Deconvolution Kernel Estimator}

One may see that to build a confidence interval for $\rho$ when measurement errors are present, the key quantities are $E(x \mid X)$ and $E(y \mid Y)$. If normality assumptions fail, these quantities are very hard to estimate. One way out is to use the deconvolution kernel technique. For more theory about this method, see Fan and Truong (1993).

For any density function $L$ symmetric around 0 , let $\phi_{L}$ denote its characteristic function, $h$ be a positive number, and define

$$
L_{h}(x)=(2 \pi)^{-1} \int_{R} \exp (-\mathbf{i} t x) \phi_{L}(t) \phi_{u}^{-1}(t / h) d t, \mathbf{i}=\sqrt{-1} .
$$

The density function $f_{x}(\cdot)$ of $x$ can be estimated by

$$
\hat{f}_{x}(t)=\frac{1}{m h} \sum_{i=1}^{m} L_{h}\left(\frac{t-X_{i}}{h}\right)
$$

Let

$$
D_{0}\left(X, f_{x}\right)=\int f_{x}(t) f_{u}(X-t) d t, \quad D_{1}\left(X, f_{x}\right)=\int t f_{x}(t) f_{u}(X-t) d t .
$$

Then $E(x \mid X)$ can be estimated by the ratio $\hat{H}_{x}(X):=D_{1}\left(X, \hat{f}_{x}\right) / D_{0}\left(X, \hat{f}_{x}\right)$. Similarly, one can estimate $E(y \mid Y)$ by the ratio $\hat{H}_{y}(Y):=D_{1}\left(Y, \hat{f}_{y}\right) / D_{0}\left(Y, \hat{f}_{y}\right)$. Under some regularity conditions, one can show that $\hat{H}_{x}(w) \rightarrow H_{x}(w), \hat{H}_{y}(w) \rightarrow H_{y}(w)$ in probability for all $w$. A point estimator of $\rho$ can be defined as the ratio $R=n \sum_{i=1}^{m} \hat{H}_{x}\left(X_{i}\right) / m \sum_{j=1}^{n} \hat{H}_{y}\left(Y_{j}\right)$. To investigate the asymptotic properties of $R$, a slight modification on the estimator $\hat{H}_{x}$ and $\hat{H}_{y}$ is needed. Let $\eta_{n}$ be a sequence of positive constants converging to 0 , and let $\hat{f}_{x, i}$ be the deconvolution 
density estimator constructed without using the $i$-th observation $X_{i}$. Define

$$
\hat{H}_{x,(i)}\left(X_{i}\right)=\frac{D_{1}\left(X_{i}, \hat{f}_{x, i}\right)}{D_{0}\left(X_{i}, \hat{f}_{x, i}\right)+\eta_{n}}, \quad \hat{H}_{y,(j)}\left(Y_{j}\right)=\frac{D_{1}\left(Y_{j}, \hat{f}_{y, j}\right)}{D_{0}\left(Y_{j}, \hat{f}_{y, j}\right)+\eta_{n}},
$$

The constants $\eta_{n}$ are a technical convenience to bound the denominator away from 0 . After imposing some conditions upon the underlying distribution of $x, y$ and the measurement errors $\xi, \eta$, we can show that, by using similar argument as in Stefanski, L.A., and Carroll, R.J. (1991),

$$
\begin{aligned}
& m E\left[\frac{1}{m} \sum_{i=1}^{m} \hat{H}_{x,(i)}\left(X_{i}\right)-\frac{1}{m} \sum_{i=1}^{m} E\left(x \mid X_{i}\right)\right]^{2} \rightarrow 0, \\
& n E\left[\frac{1}{n} \sum_{j=1}^{n} \hat{H}_{y,(j)}\left(Y_{j}\right)-\frac{1}{n} \sum_{j=1}^{n} E\left(y \mid Y_{j}\right)\right]^{2} \rightarrow 0 .
\end{aligned}
$$

The law of large numbers implies that $m^{-1} \sum_{i=1}^{m} E\left(x \mid X_{i}\right) \rightarrow E(x), n^{-1} \sum_{j=1}^{n} E\left(y \mid Y_{j}\right) \rightarrow E(y)$ in probability. Therefore,

$$
R=\frac{n \sum_{i=1}^{m} \hat{H}_{x,(i)}\left(X_{i}\right)}{m \sum_{j=1}^{n} \hat{H}_{y,(j)}\left(Y_{j}\right)} \rightarrow \frac{E(x)}{E(y)}=\rho
$$

in probability. That is, $R$ is a consistent estimator of $\rho$. Also, the central limit theorem implies that

$$
\begin{aligned}
& \sqrt{m}\left(\frac{1}{m} \sum_{i=1}^{m} E\left(x \mid X_{i}\right)-E(x)\right) \sim N(0, \operatorname{Var}(E(x \mid X))) \\
& \sqrt{n}\left(\frac{1}{n} \sum_{j=1}^{n} E\left(y \mid Y_{j}\right)-E(y)\right) \sim N(0, \operatorname{Var}(E(y \mid Y))) .
\end{aligned}
$$

Therefore, from (4.3), one has

$$
\begin{aligned}
& \sqrt{m}\left(\frac{1}{m} \sum_{i=1}^{m} H_{x,(i)}\left(X_{i}\right)-E(x)\right) \sim N(0, \operatorname{Var}(E(x \mid X))) \\
& \sqrt{n}\left(\frac{1}{n} \sum_{j=1}^{n} H_{y,(j)}\left(Y_{j}\right)-E(y)\right) \sim N(0, \operatorname{Var}(E(y \mid Y))) .
\end{aligned}
$$

Therefore, a routine argument can show that

Theorem 4.1 Suppose $m / n \rightarrow \lambda \geq 0$ as $m, n \rightarrow \infty$. Then $\sqrt{m}(R-\rho) \Longrightarrow N\left(0, \sigma_{\rho}^{2}\right)$, where

$$
\sigma_{\rho}^{2}=\frac{\operatorname{Var}(E(x \mid X))}{E^{2}(y)}+\frac{\lambda E^{2}(x) \operatorname{Var}(E(y \mid Y))}{E^{4}(y)} .
$$

Let $\hat{\sigma}_{\rho}^{2}$ be any consistent estimator of $\sigma_{\rho}^{2}$. Then a confidence interval for $\rho$ with confidence level $1-\alpha$ is given by $R \pm z_{1-\alpha / 2} \hat{\sigma}_{\rho}$, where $z_{1-\alpha / 2}$ is the $1-\alpha$ percentile of standard 
normal distribution. A simple consistent estimator of $\sigma_{\rho}^{2}$ can be obtained by replacing $E(x)$ with $\bar{X}_{m}, E(y)$ with $\bar{Y}_{m}, \operatorname{Var}(E(x \mid X))$ with the sample variance of $\hat{H}_{x}\left(X_{i}\right)$ 's, and $\operatorname{Var}(E(y \mid Y))$ with the sample variance of $\hat{H}_{y}\left(Y_{j}\right)$ 's.

If conditions (a), (b) in Section 2 hold, then one can see that Theorem 4.1 is identical to Theorem 3.1. Since $\operatorname{Var}(E(x \mid X)) \leq \operatorname{Var}(x) \leq \operatorname{Var}(\mathrm{X})$, and $\operatorname{Var}(E(y \mid Y)) \leq \operatorname{Var}(y) \leq \operatorname{Var}(\mathrm{Y})$, so the asymptotic variances of $R$ in Theorem 4.1 and Theorem 3.1 are less than that of $R$ in Theorem 2.1.

\subsection{EMPIRICAL LIKELIHOOD ESTIMATOR}

The popularity of an empirical likelihood procedure comes from its ability to combine the reliability of the nonparametric methods with the flexibility and effectiveness of the likelihood approach. An extensive introduction of this procedure can be found in Owen (2001).

First we will construct an empirical likelihood confidence interval without correcting the biases. Note that the relative potency $\rho$ in the direct bioassay is defined by $\rho=\mu_{x} / \mu_{y}$, or $\mu_{x}=\rho \mu_{y}$, therefore, we have a single estimating equation $E(X-\rho Y)=0$. If $m=n$, the profile empirical likelihood ratio function for $\rho$ can be defined as

$$
\mathscr{R}(\rho)=\max \left\{\prod_{i=1}^{n} n w_{i} \mid \sum_{i=1}^{n} w_{i}\left(X_{i}-\rho Y_{i}\right)=0, w_{i} \geq 0, \sum_{i=1}^{n} w_{i}=1\right\},
$$

and the empirical likelihood confidence interval of $\rho$ has the form of $\left\{\rho: \mathscr{R}(\beta) \geq \gamma_{0}\right\}$, where $\gamma_{0}$ depends on the asymptotic distribution of the empirical likelihood ratio function.

Usually, the sample sizes from standard and test preparations may not be the same. Even when they are same, the observations are not obtained pairwise. Under such circumstances, one should use the two-sample profile empirical likelihood ratio function

$$
\mathscr{R}(\rho)=\max \left\{\prod_{i=1}^{m} \prod_{j=1}^{n} m n w_{i j} \mid \sum_{i=1}^{m} \sum_{j=1}^{n} w_{i j}\left(X_{i}-\rho Y_{j}\right)=0, w_{i j} \geq 0, \sum_{i=1}^{m} \sum_{j=1}^{n} w_{i j}=1\right\} .
$$

Let $\rho_{0}$ to be the true relative potency. The following result states the asymptotic distribution of the two-sample empirical likelihood.

\section{Theorem 4.2 Suppose}

C1. $m, n \rightarrow \infty, m / n \rightarrow \gamma>0$,

C2. $E X^{3}<\infty, E Y^{3}<\infty, E\left(X-\rho_{0} Y\right)^{2}>0$.

Then $-2 n^{-1} K\left(\hat{\sigma}_{x}^{2}, \hat{\sigma}_{y}^{2}, \rho_{0}\right) \log \mathscr{R}\left(\rho_{0}\right) \Longrightarrow_{d} \chi_{1}^{2}$, where $K(s, t, \rho)=\left(s+\rho^{2} t\right) /\left(s+\gamma \rho^{2} t\right)$, and $\hat{\sigma}_{x}^{2}, \hat{\sigma}_{y}^{2}$ are any consistent estimators for $\sigma_{x}^{2}, \sigma_{y}^{2}$, respectively. 
Proof: Using Lagrange multipliers, let

$$
L(w ; \lambda ; \eta)=\sum_{i=1}^{m} \sum_{j=1}^{n} \log m n w_{i j}-\lambda m n \sum_{i=1}^{m} \sum_{j=1}^{n} w_{i j}\left(X_{i}-\rho_{0} Y_{j}\right)-\eta\left(\sum_{i=1}^{m} \sum_{j=1}^{n} w_{i j}-1\right),
$$

where $w=\left(w_{i j}: i=1, \ldots, m ; j=1, \ldots, n\right)$. Then, we can get

$$
w_{i j}=\frac{1}{m n} \cdot \frac{1}{1+\lambda\left(X_{i}-\rho_{0} Y_{j}\right)},
$$

where $\lambda$ satisfies

$$
g(\lambda)=\frac{1}{m n} \sum_{i=1}^{m} \sum_{j=1}^{n} \frac{X_{i}-\rho_{0} Y_{j}}{1+\lambda\left(X_{i}-\rho_{0} Y_{j}\right)}=0 .
$$

To get a bound for $\lambda$, we rewrite $g(\lambda)$ as

$$
\begin{aligned}
g(\lambda) & =\frac{1}{m n} \sum_{i=1}^{m} \sum_{j=1}^{n}\left(X_{i}-\rho_{0} Y_{j}\right)\left[1-\frac{\lambda\left(X_{i}-\rho_{0} Y_{j}\right)}{1+\lambda\left(X_{i}-\rho_{0} Y_{j}\right)}\right] \\
& =\bar{X}_{m}-\rho_{0} \bar{Y}_{n}-\lambda \frac{1}{m n} \sum_{i=1}^{m} \sum_{j=1}^{n} \frac{\left(X_{i}-\rho_{0} Y_{j}\right)^{2}}{1+\lambda\left(X_{i}-\rho_{0} Y_{j}\right)} .
\end{aligned}
$$

Denote

$$
\tilde{S}=\frac{1}{m n} \sum_{i=1}^{m} \sum_{j=1}^{n} \frac{\left(X_{i}-\rho_{0} Y_{j}\right)^{2}}{1+\lambda\left(X_{i}-\rho_{0} Y_{j}\right)}, S=\frac{1}{m n} \sum_{i=1}^{m} \sum_{j=1}^{n}\left(X_{i}-\rho_{0} Y_{j}\right)^{2} .
$$

Then from (4.6), we have

$$
\lambda \tilde{S}=\bar{X}_{m}-\rho_{0} \bar{Y}_{n} .
$$

Note that $w_{i j}>0$ for each $i$, so $1+\lambda\left(X_{i}-\rho_{0} Y_{i}\right)>0$. Therefore,

$$
|\lambda| S \leq|\lambda| \tilde{S}\left(1+|\lambda| \max \left|X_{i}-\rho_{0} Y_{j}\right|\right) .
$$

From (4.7), we have

$$
|\lambda|\left[S-\left|\bar{X}_{m}-\rho_{0} \bar{Y}_{n}\right| \cdot \max \left|X_{i}-\rho_{0} Y_{j}\right|\right] \leq\left|\bar{X}_{m}-\rho_{0} \bar{Y}_{n}\right| .
$$

By the independence of $X$ and $Y$ and the law of large numbers, one can show that $S=$ $E\left(X-\rho_{0} Y\right)^{2}+o_{p}(1)$. Since $m / n$ are asymptotically bounded, so $\bar{X}_{m}-\rho_{0} \bar{Y}_{n}=O_{p}\left(n^{-1 / 2}\right)$, and $\max \left|X_{i}-\rho_{0} Y_{j}\right| \leq \max _{1 \leq i \leq m}\left|X_{i}\right|+\rho \max _{1 \leq j \leq n}\left|Y_{j}\right|=o_{p}\left(n^{1 / 2}\right)$. Therefore, from (4.8), one has

$$
|\lambda|\left[S+o_{p}(1)\right]=O_{p}\left(n^{-1 / 2}\right)
$$

which implies $\lambda=O_{p}\left(n^{-1 / 2}\right)$ and

$$
|\lambda| \max \left|X_{i}-\rho_{0} Y_{j}\right|=O_{p}\left(n^{-1 / 2}\right) o_{p}\left(n^{1 / 2}\right)=o_{p}(1) .
$$

Note that $g(\lambda)$ defined in (4.5) can also be written as

$$
g(\lambda)=\bar{X}_{m}-\rho_{0} \bar{Y}_{n}-\lambda S-\frac{\lambda^{2}}{m n} \sum_{i=1}^{m} \sum_{j=1}^{n} \frac{\left(X_{i}-\rho_{0} Y_{j}\right)^{3}}{1+\lambda\left(X_{i}-\rho_{0} Y_{j}\right)},
$$


The last term on the right side of above equation has the order of $o_{p}\left(n^{-1 / 2}\right)$ which can be derived from the following

$$
\frac{\lambda^{2}}{m n} \sum_{i=1}^{m} \sum_{j=1}^{n} \frac{\left(X_{i}-\rho_{0} Y_{j}\right)^{3}}{1+\lambda\left(X_{i}-\rho Y_{j}\right)}=O_{p}\left(n^{-1}\right) O_{p}(1) \frac{1}{m n} \sum_{i=1}^{m} \sum_{j=1}^{n}\left(X_{i}-\rho_{0} Y_{j}\right)^{3}=O_{p}\left(n^{-1}\right) o_{p}\left(n^{1 / 2}\right) .
$$

Therefore, from (4.5),

$$
\lambda=S^{-1}\left(\bar{X}_{m}-\rho_{0} \bar{Y}_{n}\right)+\beta,
$$

where $\beta=o_{p}\left(n^{-1 / 2}\right)$. From (4.9), we have the following Taylor expansion,

$$
\log \left[1+\lambda\left(X_{i}-\rho Y_{j}\right)\right]=\lambda\left(X_{i}-\rho Y_{j}\right)-\frac{1}{2} \lambda^{2}\left(X_{i}-\rho_{0} Y_{j}\right)^{2}+\xi_{i j}
$$

where $\xi_{i j}$ 's are such that

$$
P\left(\left|\xi_{i j}\right| \leq B|\lambda|^{3}\left|X_{i}-\rho_{0} Y_{j}\right|^{3}, 1 \leq i \leq m, 1 \leq j \leq n\right) \rightarrow 1
$$

for some large enough positive $B$. Now we may write, using (4.11), (4.10)

$$
\begin{aligned}
-2 n^{-1} \log \mathscr{R}\left(\rho_{0}\right) & =-2 n^{-1} \sum_{i=1}^{m} \sum_{j=1}^{n} \log n m w_{i j}=2 n^{-1} \sum_{i=1}^{m} \sum_{j=1}^{n} \log \left[1+\lambda\left(X_{i}-\rho Y_{j}\right)\right] \\
& =2 m \lambda\left(\bar{X}_{m}-\rho_{0} \bar{Y}_{n}\right)-\lambda^{2} m S+2 n^{-1} \sum_{i=1}^{m} \sum_{j=1}^{n} \xi_{i j} \\
& =m S^{-1}\left(\bar{X}_{m}-\rho_{0} \bar{Y}_{n}\right)^{2}-m \beta^{2} S^{-1}+2 n^{-1} \sum_{i=1}^{m} \sum_{j=1}^{n} \xi_{i j}
\end{aligned}
$$

Note that

$$
\sqrt{m}\left(\bar{X}_{m}-\rho_{0} \bar{Y}_{n}\right)=\sqrt{m}\left(\bar{X}_{m}-\mu_{x}\right)-\rho_{0} \sqrt{\frac{m}{n}} \sqrt{n}\left(\bar{Y}_{n}-\mu_{y}\right) .
$$

If we let $n \rightarrow \infty$ with the independence of these two samples, the Slutsky theorem and condition C1 imply that $\sqrt{m}\left(\bar{X}_{m}-\rho_{0} \bar{Y}_{n}\right) \Longrightarrow N\left(0, \sigma_{x}^{2}+\gamma \rho_{0}^{2} \sigma_{y}^{2}\right)$. Together with the result of $S \rightarrow E\left(X-\rho_{0} Y\right)^{2}=\sigma_{x}^{2}+\rho^{2} \sigma_{y}^{2}$ almost surely, we can get

$$
m S^{-1}\left(\bar{X}_{m}-\rho_{0} \bar{Y}_{n}\right)^{2} \Longrightarrow_{d} \frac{\sigma_{x}^{2}+\gamma \rho_{0}^{2} \sigma_{y}^{2}}{\sigma_{x}^{2}+\rho_{0}^{2} \sigma_{y}^{2}} \chi_{1}^{2} .
$$

The second term in (4.12) is on the order of $o_{p}(1)$ since $\beta=o_{p}\left(n^{-1 / 2}\right), S=O_{p}(1)$, while the last term in (4.12) is negligible in probability since

$$
n^{-1}\left|\sum_{i=1}^{m} \sum_{j=1}^{n} \xi_{i j}\right| \leq n^{-1}|B||\lambda|^{3} \sum_{i=1}^{m} \sum_{j=1}^{n}\left|X_{i}-\rho_{0} Y_{j}\right|^{3}=n^{-1} O_{p}\left(n^{-3 / 2}\right) o_{p}\left(n^{5 / 2}\right)=o_{p}(1) .
$$

Therefore

$$
-2 n^{-1} \log \mathscr{R}\left(\rho_{0}\right) \Longrightarrow_{d} \frac{\sigma_{x}^{2}+\gamma \rho_{0}^{2} \sigma_{y}^{2}}{\sigma_{x}^{2}+\rho_{0}^{2} \sigma_{y}^{2}} \chi_{1}^{2} .
$$

This implies the desired result. 
In particular, if $\gamma=1$, then the theorem implies $-2 n^{-1} \log \mathscr{R}\left(\rho_{0}\right) \Longrightarrow \chi_{1}^{2}$. This is the case for balanced direct bioassay in which $m=n$.

Based on the above theory, one may construct the empirical confidence interval with confidence level $1-\alpha$ for $\rho$ as

$$
\mathscr{C}=\left\{\rho:-2 n^{-1} K\left(\hat{\sigma}_{x}^{2}, \hat{\sigma}_{y}^{2}, \rho\right) \log \mathscr{R}\left(\rho_{0}\right) \leq \chi_{1,1-\alpha}^{2}\right\},
$$

or

$$
\mathscr{C}=\left\{\frac{\sum_{i=1}^{m} w_{i j} X_{i}}{\sum_{j=1}^{n} w_{i j} Y_{j}}: \prod_{i=1}^{m} \prod_{j=1}^{n} n m w_{i j} \geq \exp \left(-\frac{n \chi_{1,1-\alpha}^{2}}{2 K\left(\hat{\sigma}_{x}^{2}, \hat{\sigma}_{y}^{2}, \rho\right)}\right), w_{i j}>0, \sum_{i=1}^{m} \sum_{j=1}^{n} w_{i j}=1 .\right\}
$$

where $\chi_{1,1-\alpha}^{2}$ is the $1-\alpha$ quantile of $\chi_{1}^{2}$.

Remark 1: Owen (2001) presents an argument for the empirical likelihood inferences in multi-sample settings, especially for two independent samples case. Adapting his procedure to our case, the following profile empirical likelihood ratio function shall be used

$$
\mathscr{R}(\rho)=\max \left\{\prod_{i=1}^{m} \prod_{j=1}^{n} m n u_{i} v_{j} \mid \sum_{i=1}^{m} \sum_{j=1}^{n} u_{i} v_{j}\left(X_{i}-\rho Y_{j}\right)=0, u_{i}, v_{j} \geq 0, \sum_{i=1}^{m} u_{i}=\sum_{j=1}^{n} v_{j}=1\right\} .
$$

Because of the independence, the above profile empirical likelihood ratio function seems more reasonable and natural. Based on an ANOVA decomposition technique, one can expect that $-2 \log \mathscr{R}\left(\rho_{0}\right) \Longrightarrow \chi_{1}^{2}$. Another merit of his argument is that one only requires $\min (m, n) \rightarrow \infty$, and there is no need to have them grow at the same rate. But Owen's (2001) argument is nonrigorous, some other conditions may needed to guarantee a smooth flow of proof. For example, to get a legitimate expansion for $u_{i}$ and $v_{j}$, one needs to find a bound for $\lambda$, which is not trivial. Here we developed a new empirical likelihood procedure for our needs without considering independence when constructing the profile empirical likelihood ratio function, but a rigorous and typical argument can be made to obtain the asymptotic distribution of $-2 \log \mathscr{R}\left(\rho_{0}\right)$. Finally, it is worth mentioning that, unlike other typical asymptotic results, we have an extra normalizing factor $n^{-1} K\left(\hat{\sigma}_{x}^{2}, \hat{\sigma}_{y}^{2}, \rho\right)$ in front of $-2 \log \mathscr{R}\left(\rho_{0}\right)$.

Remark 2: It is well known that the computation for the empirical likelihood is very extensive. A slight modification on the above procedure can lead to simpler theory, moreover, we can borrow available programs to implement the empirical likelihood procedure. The modified version considers the following profile empirical likelihood ratio:

$$
\mathscr{R}(\rho)=\max \left\{\prod_{i=1}^{m} m w_{i} \mid \sum_{i=1}^{m} w_{i}\left(X_{i}-\rho \bar{Y}_{n}\right)=0, w_{i} \geq 0, \sum_{i=1}^{m} w_{i}=1\right\} .
$$

Slightly modification on the above argument leads to a similar result as in Theorem 4.2 which is summarized in the following theorem. 


\section{Theorem 4.3 Suppose}

C1. $m, n \rightarrow \infty, m / n \rightarrow \gamma>0$,

C2. $E X^{3}<\infty, E Y^{3}<\infty$ and $E\left(X-\rho_{0} Y\right)^{2}>0$.

Then $-2 K\left(\hat{\sigma}_{x}^{2}, \hat{\sigma}_{y}^{2}, \rho_{0}\right) \log \mathscr{R}\left(\rho_{0}\right) \Longrightarrow_{d} \chi_{1}^{2}$, where $K(s, t, \rho)=s /\left(s+\gamma \rho^{2} t\right)$, and $\hat{\sigma}_{x}^{2}$, $\hat{\sigma}_{y}^{2}$ are any consistent estimators for $\sigma_{x}^{2}, \sigma_{y}^{2}$, respectively.

Finally, we will use the SIMEX technique to remove the effect resulting from the measurement errors. It has two steps:

- Simulation Step: For $\lambda \geq 0$, generate an i.i.d. sample of size $m$ from the distribution of $u$, say $\tilde{u}_{i}, i=1,2, \ldots, m$, calculate $\tilde{X}_{i}=X_{i}+\sqrt{\lambda} \tilde{u}_{i}$; generate an i.i.d. sample of size $n$ from the distribution of $v$, say $\tilde{v}_{j}, j=1,2, \ldots, n$, calculate $\tilde{Y}_{j}=Y_{j}+\sqrt{\lambda} \tilde{v}_{j}$. Then calculate the empirical likelihood confidence interval using $\tilde{X}_{i}$ 's and $\tilde{Y}_{j}$ 's and formula (4.13). Repeat the above $B$ times to obtain $B$ confidence intervals, then calculate the average of the left end points, denoted by $L(\lambda)$, and the average of the right end points, denoted by $R(\lambda)$. Redo the above step by using different $\lambda \geq 0$. Suppose we are selecting $\lambda=\lambda_{1}, \lambda_{2}, \ldots, \lambda_{k}$. Then we obtain $L\left(\lambda_{1}\right), L\left(\lambda_{2}\right), \ldots, L\left(\lambda_{k}\right)$, and $R\left(\lambda_{1}\right), R\left(\lambda_{2}\right), \ldots, R\left(\lambda_{k}\right)$, Finally, using a quadratic, cubic or other proper curve to fit the data set $L\left(\lambda_{k}\right)$ 's and $R(\lambda)$ 's, respectively.

- Extrapolation step: Extrapolate the fitted curves to $\lambda=-1$. Denote the fitted value as $L(-1)$ and $R(-1)$. Then the SIMEX empirical likelihood confidence interval for the relative potency is given by $(L(-1), R(-1))$.

\section{SIMULATION STUDIES}

To illustrate the performance of the proposed estimating procedures, some simulation studied are described in this section. The data were generated according to $x \sim N(2,1)$, $y \sim N(1,1), u \sim N\left(0, \sigma_{u}^{2}\right), v \sim N\left(0, \sigma_{v}^{2}\right), X=x+u, Y=y+v$, so the true relative potency is 2 . The sample sizes in the simulation were chosen to be 50,100, 200 and 500. To see the effect of the variability of measurement error on the inference results, we chose $\sigma_{u}^{2}=\sigma_{v}^{2}=0.1,0.3$ and 0.5. Each estimating procedure was repeated 500 times, the mean and the standard deviation of the resulting estimates for the relative potency are presented in the following table, where MSE denotes the mean square error of the estimator, Cover.Rt stands for the simulated coverage rate of the confidence interval, and Length shows the average length of the confidence interval. The confidence level is chosen to be 0.95 across all estimating procedures.

As we expected, when the variance of the measurement error increases, the MSEs of all estimators also increase. The confidence intervals from naive estimating procedures hold 


\begin{tabular}{c|c|ccc|ccc|ccc}
\hline \hline & & \multicolumn{3}{c|}{$\sigma^{2}=0.1$} & \multicolumn{3}{c|}{$\sigma^{2}=0.3$} & \multicolumn{3}{c}{$\sigma^{2}=0.5$} \\
\hline & $\mathrm{n}$ & MSE & Cover.Rt & Length & MSE & Cover.Rt & Length & MSE & Cover.Rt & Length \\
\hline \multirow{5}{*}{ Naive } & 50 & 0.1687 & 0.946 & 1.5793 & 0.2404 & 0.946 & 1.5847 & 0.3382 & 0.944 & 1.8817 \\
& 100 & 0.0661 & 0.934 & 0.9913 & 0.0799 & 0.946 & 1.0958 & 0.0938 & 0.948 & 1.1967 \\
& 200 & 0.0267 & 0.950 & 0.6687 & 0.0318 & 0.944 & 0.7321 & 0.0370 & 0.950 & 0.7920 \\
& 500 & 0.0122 & 0.940 & 0.4183 & 0.0146 & 0.944 & 0.4563 & 0.0169 & 0.944 & 0.4916 \\
\hline \multirow{5}{*}{ Bias.Cr } & 50 & - & 0.928 & 1.3940 & - & 0.856 & 1.3395 & - & 0.790 & 1.5027 \\
& 100 & - & 0.904 & 0.8934 & - & 0.852 & 0.8233 & - & 0.782 & 0.7687 \\
& 200 & - & 0.932 & 0.6054 & - & 0.864 & 0.5566 & - & 0.814 & 0.5182 \\
& 500 & - & 0.928 & 0.3797 & - & 0.850 & 0.3494 & - & 0.790 & 0.3255 \\
\hline \multirow{5}{*}{ Decon. } & 50 & 0.1264 & 0.934 & 1.2738 & 0.1647 & 0.902 & 1.2515 & 0.2046 & 0.864 & 1.2411 \\
& 100 & 0.0600 & 0.930 & 0.8710 & 0.0701 & 0.888 & 0.8364 & 0.0816 & 0.866 & 0.8119 \\
& 200 & 0.0301 & 0.918 & 0.6123 & 0.0361 & 0.884 & 0.5829 & 0.0418 & 0.834 & 0.5606 \\
& 500 & 0.0113 & 0.910 & 0.3776 & 0.0139 & 0.872 & 0.3559 & 0.0175 & 0.828 & 0.3465 \\
\hline \multirow{5}{*}{ SIMEX } & 50 & 0.1209 & 0.960 & 1.4005 & 0.1487 & 0.962 & 1.5265 & 0.1797 & 0.956 & 1.6463 \\
& 100 & 0.0598 & 0.950 & 0.9363 & 0.0714 & 0.944 & 1.0159 & 0.0830 & 0.942 & 1.0896 \\
& 200 & 0.0299 & 0.938 & 0.6602 & 0.0348 & 0.938 & 0.7164 & 0.0399 & 0.930 & 0.7686 \\
& 500 & 0.0118 & 0.952 & 0.4154 & 0.0146 & 0.956 & 0.4528 & 0.0173 & 0.946 & 0.4874 \\
\hline \multirow{5}{*}{ EL-SIMEX } & 50 & 0.1131 & 0.938 & 1.2644 & 0.1348 & 0.900 & 1.2764 & 0.1595 & 0.866 & 1.3013 \\
& 100 & 0.0625 & 0.930 & 0.9545 & 0.0776 & 0.890 & 0.9694 & 0.0922 & 0.874 & 0.9925 \\
& 200 & 0.0282 & 0.944 & 0.6588 & 0.0319 & 0.942 & 0.6620 & 0.0359 & 0.916 & 0.6710 \\
& 500 & 0.0129 & 0.912 & 0.4012 & 0.0155 & 0.878 & 0.4053 & 0.0179 & 0.846 & 0.4066 \\
\hline \multirow{5}{*}{} & & & & & & & & & &
\end{tabular}

the nominal confidence level very well. Although the confidence intervals from the bias corrected estimating procedure has a much smaller length, it severely underestimates the nominal confidence, which supports our discussion in Section 3. It is well known that the Deconvolution procedure is less effective than it should be, this point is again confirmed by our simulation. Compared to the naive estimating procedure, the confidence intervals are narrower, but the simulated coverage rate, although a little bit higher than the bias correction method, is still underestimated. The SIMEX method really stands out from all other estimating procedures in that it not only keeps the nominal confidence, but also has a narrower confidence interval than the naive one. To expedite the computation, we use the SIMEX procedure based on Theorem 4.3. We also know that the SIMEX method performs better when the measurement error follows normal distribution, some simulations are also done for other distribution families, our findings indicate that the SIMEX still works very well for symmetric distribution families. This implies the robustness of the SIMEX procedure. Similar to other nonparametric methods, the estimates from EL-SIMEX procedure have small mean square errors, but the empirical confidence levels are far below the nominal 0.95 level.

As far as the confidence levels are concerned, the naive estimation procedure works consistently well for all scenarios. Its MSEs are a little bit larger for small sample sizes, but they eventually become smaller, comparable to other nonparametric methods. So, if the sample size in a direct bioassay is large, we recommend using the naive estimator to estimate the relative potency. But if the sample size is small which is often the case in real direct bioassays, then one of the above mentioned nonparametric procedures, in particular, the SIMEX estimator, should be used. 
We also conducted a simulation study using naive method, bias correction method and SIMEX method when the measurement errors follow a log-normal distribution. The naive method worked well. Surprisingly, the SIMEX method still keeps its goodness. We did not consider the deconvolution method, since no explicit expression of deconvolution kernel is available for this case, and some complicate numerical integration might be necessary. We will evaluate the performance of this method in the future once an efficient algorithm is found. But we expect the deconvolution method gives a better result.

Acknowledgement. The author is grateful to the anonymous referee for providing a constructive criticism that helped to improve the presentation of the paper.

\section{REFERENCES}

Carroll, R.J., Küchenhoff,H., Lombard,F. and Stefanski L.A. (1996), “ Asymptotics for the SIMEX Estimator in Nonlinear Measurement Error Models”. JASA 91, 242250.

Carroll, R.J., Ruppert, D. and Stefanski, L.A. (1995). Measurement Error in Nonlinear Models, Chapman \& Hall/CRC, Boca Raton.

Cook, J. \& Stefanski, L.A. (1995). “A simulation extrapolation method for parametric measurement error models", JASA 89, 1314-1328.

Fan,J. \& Truong, K.T. (1993). Nonparametric regression with errors in variables. Ann. Statist. 21 1900-1925.

Ferguson, T.S. (1996), A course in large sample theory, Chapman \& Hall/CRC.

Fieller, E.C. (1932), "The distribution of the index in a normal bivariate population", Biometrika. 24 428-440.

Finney, D.J. (1978), Statistical Methods in Biological Assay, 3rd edition, London: Griffin.

Fuller, W.A. (1987). Measurement Error Models.Wiley, New York.

Govindarajulu, Z. (2001), Statistical Techniques in Bioassay, 2nd edition, Karger.

Hubert, J.J. (1992), Bioassay, 3rd edition, Kendall/Hunt Publishing Company.

Owen,A.B. (2001), Empirical Likelihood. Chapman \& Hall/CRC.

Stefanski, L.A., and Carroll, R.J. (1991). Deconvolution-based score tests in measurement error models. The Annals of Statistics 19 249-259.

Stefanski, L.A. and Cook, J. (1995). "Simulation extrapolation: the measurement error jackknife", JASA, 90, 1247-1256 